\title{
Libre accès à l'information scientifique et technique : état de l'art et perspectives
}

Organisé à Paris les 23 et 24 janvier derniers par le ministère de la Recherche, I'INSERM, le CNRS, I'INIST et I'ICSTI, un séminaire international s'est penché sur les conditions, les modalités et les perspectives de l'accès ouvert à l'information scientifique et technique : quelles conséquences pour la diffusion de I'IST ? Quels enjeux pour la communauté scientifique? Quelle opportunité pour les pays en développement? La présentation de plusieurs projets et initiatives a étayé les réflexions sur les modèles techniques et économiques à concevoir.

L'ACCES OUVERT AUX TRAVAUX ET PUBLICATIONS SCIENTIFIQUES ELECTRONIQUES, actuellement sujet de nombreux débats dans les communautés des chercheurs, des éditeurs et des professionnels de l'information et documentation, représente un enjeu capital pour la diffusion des connaissances. Les différents comptes rendus d'expériences présentés lors de ces deux journées, suivies par deux cent cinquante personnes venues de trente pays, devaient permettre de dresser un "état de l'art du libre accès » et d'organiser la réflexion, avec une attention particulière aux besoins des pays en développement, sur des modèles qui ne sont de loin pas encore stabilisés, en particulier en matière de coûts ou d'usages.

Nous proposons dans ce compte rendu des synthèses des interventions et débats qui ont eu lieu au cours de cette manifestation - interventions dont la liste, les titres et les auteurs sont précisés en hors texte pages 38 à 41.

\section{Vue d'ensemble}

En ouverture, Christine Deschamps, présidente en exercice de I'IFLA, a mis l'accent sur le rôle joué par les professionnels de l'information ou infomédiaires dans les négociations en cours pour obtenir un consensus sur un modèle d'accès équitable et durable à une information de qualité et de prix raisonnable, selon les normes bibliothéconomiques ${ }^{1}$.

Kurt Molholm a ensuite montré le rôle important que joue l'ICSTI pour faire reconnaître la valeur de l'information scientifique et technique et les actions qu'il mène, en collaboration avec divers organismes tels que l'Unesco, en faveur de la conservation de cette information. Selon I'ICSTI, I'IST doit faire partie du « domaine public » et de nouveaux modèles doivent être promus, qui prennent en compte les problèmes liés aux règles de droit d'auteur et aux dérives que les possibilités de contrôle technique rendent envisageables (comme par exemple l'installation de protections par les ayants droit). Il convient d'en analyser précisément les aspects techniques et

\footnotetext{
${ }^{1}$ L'IFLA négocie depuis plusieurs mois avec I'International Publisher Associations. Elle présente également son point de vue dans le cadre des négociations en cours au sein de l'OMC.
} 
économiques à la lumière des expériences déjà engagées et des initiatives déjà prises en matière d'accès ouvert.

Avant d'évoquer l'expérience de l'INSERM, Pierre Oudet a insisté sur les points clés développés dans le rapport récemment rédigé par Jack Franklin pour l'Inist (voir plus loin) : le coût sans cesse croissant de l'accès aux publications scientifiques, la nécessaire adaptation des systèmes d'évaluation à la diffusion de la connaissance (l'évaluation réelle se faisant a posteriori), les méthodes permettant de faire face à l'accroissement exponentiel des informations (des hyperliens pertinents, par exemple, permettent de gérer la masse d'informations), l'apparition de nouvelles professions comme celle d'ingénieur bio-informatique, gestionnaire des données factuelles dans le domaine biologique ${ }^{2}$.

Le modèle de gestion de prépublications développé par I'INSERM consiste à centraliser le texte intégral des articles, puis à les indexer et à établir divers liens, entre autres avec les sites des éditeurs ou avec diverses bases de données factuelles. L'institut se charge aussi d'en assurer la pérennité. Le système mis en place permet d'identifier des compétences et des types de collaboration existants ou susceptibles d'être créés. Il sert ainsi de matière première à différentes réflexions, menées en collaboration avec des organismes européens et américains, destinées à accroître la performance de cet outil.

Pour clore la séquence d'introduction de ce séminaire, le sociologue Dominique Wolton a mis l'accent sur le changement de statut de l'information dont la valeur économique prend de plus en plus d'ampleur et sur la nature culturelle, plus que technique, de cette révolution. Ce que l'on appelle "société de l'information » consiste en la multiplication des canaux d'information, qui n'a pas forcément d'incidence sur la qualité de la communication. En outre, l'idéal que représente le partage de l'information scientifique constitue aussi un enjeu économique. La science occupe ainsi une position ambiguë et il faut aujourd'hui passer du stade de la «fascination» pour les nouvelles technologies à la réflexion sur ce que représente l'économie du savoir face à la notion de « domaine public », sur le glissement nécessaire de l'économique vers le politique et la notion d'intérêt général.

Il a ensuite proposé quelques pistes de réflexion, rappelant qu'il existe trois types d'information (la presse, la connaissance et les services), trois groupes de métiers chargés de la valorisation de l'information (les professionnels de l'information, les journalistes et le monde académique) et trois chantiers qui, à son avis, doivent être abordés. Ils visent à supprimer toute opposition entre anciens et nouveaux médias - car, si les techniques sont nouvelles, les problèmes posés sont anciens -, à éviter de succomber à la fascination technique et aux mirages économiques, et à mener une réflexion en amont sur l'intérêt général au niveau mondial, ce qui implique une valorisation des infomédiaires (humains) qui savent hiérarchiser l'information et qui, à ce titre, sont plus que jamais nécessaires.

Dominique Wolton conclut en affirmant que l'information est un enjeu politique majeur, au même titre que la santé ou l'écologie, en rappelant aussi la responsabilité des scientifiques qui doivent éviter tout corporatisme dans leurs réflexions, ainsi que la nécessité d'une ouverture des sciences « dures» à d'autres sciences, en particulier aux sciences sociales appelées à jouer un

\footnotetext{
${ }^{2}$ Un nouveau profil de bibliothécaire qui, selon Pierre Oudet, aurait pour effet d'élargir le champ de cette profession qui auparavant ne fournissait que des références bibliographiques! Il lui appartiendrait aujourd'hui de gérer du texte intégral, des données complémentaires, des bases de données factuelles et des outils de plus en plus lourds permettant de traiter ces données. Ce qui nous semble être sa fonction depuis de nombreuses années déjà et ne nous apparaît pas comme une innovation récente!...
} 
rôle fondamental dans la société de l'information, ne serait-ce qu'en matière de diversité linguistique ou culturelle.

\section{L'état de l'art}

- Une nouvelle intermédiation. Fondant sa réflexion sur un récent colloque ${ }^{3}$, Paul Uhlir affirme qu'il ne doit pas y avoir de barrière à l'accès à l'information dans le domaine scientifique. La recherche publique, qui s'appuie sur des normes et des valeurs antérieures à l'ère numérique, est un système ouvert et communautaire dont les résultats sont d'intérêt général. Dans l'environnement numérique, le domaine public s'est rétréci par l'accroissement de la pression économique et technique des propriétaires de droits, ce qui conduit à réexaminer la valeur et les limites de I'IST dans ce domaine. L'information du secteur public doit néanmoins appartenir au domaine public, pour plusieurs raisons : il n'y a pas d'incitation par la propriété intellectuelle, elle est financée par les impôts, sa circulation correspond à des valeurs démocratiques et, bien que ce soit indirectement et difficilement quantifiable, elle contribue au bien-être des populations.

Si certaines informations du secteur public ont une diffusion limitée, par exemple pour des raisons de sécurité nationale ou de respect de la vie privée, ce ne doit pas être le cas de l'information scientifique qui a des caractéristiques particulières dues à sa spécialisation extrême et à ses normes spécifiques, et qui ne peut pas dépendre directement des lois du marché. La science, en effet, doit être financée par des fonds publics car les résultats obtenus $n$ 'ont pas toujours des applications immédiates. Le large accès aux informations scientifiques permet, en revanche, des applications commerciales ultérieures par des entreprises qui n'auraient pas vu l'intérêt d'investir dans la recherche. De ce fait, l'IST doit avoir la visibilité la plus large possible, être disponible au coût le plus bas et permettre des modes multiples de réutilisation. L'information a une nature particulière puisqu'elle ne s'épuise pas par la consommation, mais au contraire en acquiert davantage de valeur. Enfin l'incitation dans le domaine scientifique ne peut pas être directement financière et le recours au droit d'auteur ne doit garantir que le respect de l'intégrité et la reconnaissance.

Dans une deuxième partie, Paul Uhlir constate l'apparition d'une nouvelle intermédiation et le passage, dans l'environnement électronique, d'une propriété intellectuelle à une communauté des biens intellectuels - réponse au rétrécissement du domaine public. De multiples expérimentations, fondées sur la coopération, l'ouverture et le partage de l'information, ont eu lieu sur le réseau, impliquant un changement dans la chaîne de l'information: de multiples différences existent entre le modèle de la publication sur support papier et le modèle ouvert du réseau numérique. Les facteurs clés de succès ne sont de ce fait plus les mêmes: dans la production et la distribution traditionnelles de biens, ils étaient fondés sur l'économie de marché, les mécanismes hiérarchisés et des droits de propriété intellectuelle pour contrôler les ressources; dans l'environnement numérique, la première réaction des éditeurs a consisté, de ce fait, à augmenter le contrôle légal et technique. Mais parallèlement sont apparus des modèles proposant des documents en libre accès, y compris des documents protégés par le droit d'auteur, présentés pour différentes raisons, entre autres à des fins «publicitaires», et constituant une nouvelle forme de production et de diffusion dans les secteurs public et privé.

\footnotetext{
${ }^{3}$ Symposium sur le rôle des données et informations scientifiques et techniques dans le domaine public, organisé en septembre 2002 par l'Académie nationale des États-Unis (et dont les réflexions concernaient uniquement ce pays). 〈www.codata.org/codata.canada
} 
Cette mise à disposition indépendante du marché, d'une organisation donnée ou d'une propriété de l'information, représente une forme partagée de production et de diffusion de l'information. La rétribution n'est plus assurée directement par des droits d'auteurs mais indirectement par la notoriété acquise. Ce modèle, fondé sur le volontariat, suppose néanmoins l'adhésion à certaines normes de fonctionnement, l'existence d'un groupe central de collaborateurs, la réciprocité, la coopération, l'autonomie. Et il offre de nouvelles opportunités de collaboration, de nouvelles intermédiations en dehors d'une institution donnée, n'obéissant pas aux normes des mécanismes de marché classiques adoptés pour le support papier: une approche dynamique dont les mécanismes devront être approfondis lors d'études et de séminaires à venir.

- Un rapport sur l'évolution du libre accès et ses conséquences. Jack Franklin a été chargé par l'Inist d'une étude visant à « faire ressortir les principaux problèmes liés aux évolutions des technologies du libre accès et à leur adoption ${ }^{4}$. Au départ, la mésentente avec le secteur de l'édition était essentielle dans ce mouvement qui, pour de nombreux scientifiques, est perçu comme devant révolutionner leur mode de travail. Mais d'autres problèmes également importants restent à résoudre, comme celui de la conservation. Le grand nombre d'expériences déjà faites ne constitue cependant qu'un point de départ. La validation des publications reste nécessaire, de même que la valeur ajoutée éditoriale (comme par exemple la mise en visibilité des différentes versions d'un article qui ne peut pas être assurée par la technique, aussi sophistiquée soit-elle). D'ailleurs, si plusieurs réalisations, comme celles qui concernent les liens croisés (autre exemple de valeur ajoutée), sont remarquables, elles restent malheureusement onéreuses. Les derniers chapitres de ce rapport concernent les licences, les coûts, l'apport pour les pays en développement et l'avenir. L'auteur a précisé que, si le libre accès est intéressant intellectuellement, il n'apparaît pas forcément comme la solution la moins coûteuse.

- Aspects juridiques. Dans le domaine du droit, abordé ensuite, Thomas Dreier souligne la confusion qui est souvent faite entre les éléments du cadre juridique, les mesures de protection technique et la gestion des droits. Mais la loi protège aussi les investissements qui s'avèrent de plus en plus souvent nécessaires. Le droit d'auteur permet de générer des profits mais il peut répondre à d'autres incitations, comme la notoriété.

Au nom du droit, il est également possible de bloquer les accès à l'information. La directive sur les bases de données $a$, d'une certaine manière, confirmé la protection des investissements dans la création et cette protection des accès par des licences. Mais des protections techniques peuvent imposer des barrières aux cuures ou informations non protégées, du seul fait qu'elles exigent un investissement particulier. La protection des données ne doit répondre qu'aux besoins de la lutte contre la piraterie, il convient donc de différencier les niveaux de protection en évitant les effets de verrouillage susceptibles de bloquer aussi les actes permis par la loi. Puisque les contraintes financières ne doivent pas être négligées et que les universités rencontrent aujourd'hui des problèmes budgétaires, il n'est pas exclu qu'elles envisagent, à leur tour, d'être des producteurs et qu'elles vendent leurs publications.

Influencé par la technique et l'économie, le copyright a instauré une protection sans doute excessive, mais il n'est pas responsable de tous les problèmes liés à la politique d'information.

\footnotetext{
${ }^{4}$ Le libre accès à l'information scientifique et technique : état de l'art, rapport général rédigé pour I'INIST par Jack Franklin, ASFRA bv, Edam, Pays-Bas, novembre 2002. Accessible intégralement à l'adresse <www.inist.fr/openaccess/fr/etat_art.php〉. Présentant les grandes lignes de ce rapport, l'auteur a souligné que les informations rassemblées, datées de novembre 2002, avaient déjà beaucoup évolué.
} 
- L'initiative pour les archives ouvertes. L'exposé d'Andy Powell, qui représente une structure chargée de conseiller les bibliothèques britanniques en matière de numérisation (UKOLN), est plus technique: il s'agit en effet de présenter le protocole Open Archives Initiative qui concerne la collecte des métadonnées. L'OAI est né de la nécessité de disposer d'une interface unique pour effectuer des recherches dans divers serveurs d'auto-archivage, qu'ils soient ou non en libre accès. L'intervenant a détaillé les points sur lesquels un accord s'est avéré nécessaire, et présenté les différences majeures entre les versions successives du protocole auquel ses initiateurs tiennent cependant à conserver une certaine stabilité pour ne pas en décourager l'usage. Dix points très techniques ont aussi été précisés, mettant l'accent sur son faible coût, sa facilité d'utilisation, sa flexibilité et sa rapidité d'exécution.

Enfin les conséquences du recours à ce protocole pour les institutions, les bibliothèques et les chercheurs ont été évoquées, pour démontrer surtout l'élément perturbateur, au sens positif du terme, qu'il a pu représenter, obligeant à repenser le mode de fonctionnement et à clarifier, voire redéfinir, le rôle de chacun. En effet, si l'utilisation de cet outil ne pose pas de problème majeur, il en pose bien plus lorsqu'il s'agit d'initier un changement culturel, poussant à innover dans la proposition de services.

\section{Des points de vue}

- Un éditeur. Le groupe Elsevier souligne son intérêt pour l'archivage libre qui améliore l'accessibilité à la littérature scientifique. Son représentant estime, en revanche, que nous vivons une situation de transition, de désordre, qui précède la mise en place d'un nouveau modèle. Les éditeurs tendent aujourd' hui à recourir à la licence pour donner accès aux publications, alors que parallèlement se mettent en place diverses initiatives pour les archives ouvertes. Quel est le système qui sera le plus attractif, étant donné que l'une des valeurs essentielles d'une revue est l'examen par les pairs? Peu importe qui va payer - l'auteur, son institution ou le lecteur. $L$ 'archivage, en revanche, incombe aux bibliothèques et Elsevier vient, à cette fin, de conclure un accord avec la Bibliothèque nationale des Pays-Bas. La seule menace réelle, c'est l'absence d'accès à une revue qui cesse alors d'être concurrentielle. Elsevier a su s'adapter grâce à des économies d'échelle. Les divers exemples évoqués ensuite en matière de libre accès confirmeraient que le modèle traditionnel, proposé par les éditeurs, semble encore répondre à de nombreuses attentes, pour des raisons financières et parce qu'il présente de réelles incitations pour les auteurs.

- Les éditeurs de revues savantes. La représentante de I'ALPSP, une association d'éditeurs de revues, rappelle qu'éditeurs et auteurs poursuivent le même objectif: donner la meilleure visibilité possible aux publications, ce pour quoi les nouvelles technologies offrent maintes opportunités. Ces dernières années, les éditeurs ont déjà proposé plusieurs solutions permettant, par le biais des licences proposées à des consortiums, d'élargir les accès à différents niveaux. Plus souples que par le passé, ces licences peuvent admettre désormais certains usages, comme les utilisations pédagogiques, le prêt entre bibliothèques ou l'archivage. Le libre accès est une opportunité qui a intéressé plusieurs éditeurs, partenaires de divers programmes permettant, entre autres, une meilleure diffusion dans les pays en développement et un soutien aux auteurs locaux.

Les coûts, néanmoins, restent bien présents dans ce nouvel environnement, qu'il s'agisse de coûts directs ou indirects, plus difficiles à évaluer. Tout aussi délicate est la nécessité d'évaluer l'impact de la gratuité sur la qualité des publications et les modes de circulation de l'information. Les éditeurs n'ont pas su présenter toute la valeur ajoutée qu'ils apportent au processus. Le bénéfice, en outre, reste un élément indispensable. Il doit être réinvesti pour améliorer les 
performances et pour soutenir différentes activités liées à la recherche. Si le libre accès est un modèle séduisant, il pose encore, en cette période intermédiaire, plusieurs questions sur le modèle économique qu'il doit représenter.

- Un éditeur de publications en libre accès. L'éditeur BioMed Central souligne aussi que le libre échange des résultats de la recherche s'impose dans le domaine scientifique, qu'il doit se traduire par un accès gratuit et ouvert favorisé par l'Internet. Dans ce nouvel environnement, le modèle économique fondé sur des abonnements et des licences n'est pas le modèle adapté aux besoins des chercheurs. BioMed Central propose des revues en libre accès dont les articles sont validés par des comités d'experts, des archives ouvertes et des données accessibles de manière pérenne, sous un format ad hoc, lisibles de diverses manières. Ce modèle renverse ainsi le modèle classique puisque le financement se fait en amont, par les institutions et non par les auteurs, ainsi que par la publicité. Le libre accès aux publications leur donne une meilleure visibilité et un meilleur impact, les délais de publication sont plus courts et les coûts pour la communauté inférieurs.

- Les agrégateurs. Un fournisseur de services aux bibliothèques, intermédiaire commercial entre les éditeurs et les utilisateurs, constate que les positions des uns et des autres se sont progressivement rejointes. Mais il remarque également que le libre accès pose des problèmes aux bibliothèques qui, faute de ressources technologiques, ne peuvent pas assurer la maintenance de ces archives. Ce sont les agrégateurs qui peuvent les aider dans cette tâche. L'approche actuelle de la base de données reste très traditionnelle, proche de la base de données factuelles, alors qu'elle est très différente dans le domaine de l'édition. Contrairement à ce qui est souvent affirmé, les coûts marginaux, dans l'environnement électronique, ne sont pas nuls: que le protocole OAI doit être amélioré, en permettant une plus grande souplesse dans le contrôle des accès et dans la tarification, pour pouvoir agréger les droits d'un individu, et faire baisser les coûts. Il faut aussi rappeler que la notion de sources ouvertes ne signifie ni liberté totale, ni gratuité totale, mais accessibilité a priori à la communauté à laquelle elles sont destinées, et que, si de nombreuses expérimentations ont eu lieu, plusieurs ont échoué ; les raisons de ces échecs doivent être analysées.

- Les bibliothèques. Le point de vue des bibliothèques est présenté par un animateur du groupe de Francfort ${ }^{5}$ et d'une association de bibliothèques de recherche (LIBER). L'évocation historique de l'évolution des bibliothèques sert de prétexte à rappeler leur rôle qui consiste non seulement à fournir les documents recherchés par divers usagers mais également à donner des informations sur leur contexte, ce qui correspond à ce que l'on appelle désormais le document profond (deep web) et le lien croisé. Elles jouent aussi un rôle fondamental dans la chaîne de l'information scientifique par l'acquisition des publications et dans leur mission de conservation. La numérisation ne représente, pour elles aussi, qu'une opportunité nouvelle pour remplir ces missions traditionnelles. En revanche, les coûts croissants des publications, qui menacent également les petits éditeurs, ont conduit à étudier des systèmes alternatifs. Si l'accès ne peut pas être gratuit, il convient d'étudier divers modèles économiques, mais en collaboration avec diverses institutions et métiers, pour mettre au point des systèmes - dont le libre accès n'est qu'un des paramètres - orientés en fonction des usages et non des institutions et qui soient en phase avec de nouvelles pratiques.

- Les chercheurs. Stevan Harnard rappelle le souci de visibilité qui est celui des chercheurs pour leurs publications et l'apport du libre accès en terme d'impact. Mais le libre accès, affirme-

\footnotetext{
${ }^{5}$ Le Groupe de Francfort est un groupe informel d'acteurs de la chaîne de la publication qui
} tente d'obtenir des consensus sur différents sujets de préoccupation communs à ses membres. 
t-il en revanche, est un mouvement qui ne concurrence pas l'édition classique. Il ne doit pas être perçu comme un moyen de résoudre des problèmes budgétaires (même s'il y contribue), ni comme un moyen de donner un accès aux enseignants, aux étudiants et au grand public, ni comme une solution à tous les problèmes d'accès des pays en développement. Ce n'est qu'un moyen d'accélérer le cycle scientifique permettant à un public de chercheurs considérablement élargi, au niveau mondial, de s'appuyer plus rapidement sur les résultats des recherches précédentes.

Les chercheurs sont également à l'origine de l'« Initiative de Budapest pour l'accès ouvert aux publications $»^{6}$. Ce mouvement, suscité par l'Open Society Institute ${ }^{7}$, essaie de remédier aux coûts excessifs des revues scientifiques. La première solution apportée, qui consistait en la création de consortiums de bibliothèques et en la négociation de licences de sites, n'a permis que de «gagner du temps»: les premières réductions de budget ont recréé les conditions du cercle vicieux que l'on avait connu précédemment (comme les consortiums avaient été subventionnés, les restrictions budgétaires ont ranimé la spirale inflationniste et provoqué de nouvelles suppressions d'abonnements). Le libre accès, fondé sur deux stratégies complémentaires - l'autoarchivage et les revues en libre accès -, créerait une situation à la fois plus satisfaisante et pérenne, évitant, en outre, "l'astigmatisme du paysage scientifique» que créent les consortiums.

Actuellement des études et des projets sont en cours pour « reconstruire l'ensemble du processus de publication » et remédier à toutes formes d'exclusion, notamment celles des pays en développement et des chercheurs « non reconnus». Il s'agit aussi de restaurer une continuité dans la progression hiérarchique, en supprimant les effets pervers induits par le système de citation et le verrouillage des accès dus au prix élevé des publications qui ont le plus fort impact. Il faut aussi souligner que, paradoxalement, de nombreuses fondations, résultats de réussites capitalistiques, collaborent en ce moment pour lutter contre la perte de l'ensemble du patrimoine, consécutive à la privatisation croissante de la propriété intellectuelle, et que les réflexions doivent aussi permettre de dresser une «carte géopolitique du savoir » plus proche de la réalité, permettant d'effectuer, à meilleur escient, des choix nationaux.

\section{Des réalisations concrètes}

- Le programme NUMDAM. Ce programme de numérisation de documents anciens mathématiques, géré par la cellule MathDoc du CNRS, répond au double souci de conservation du patrimoine culturel mathématique, en particulier du patrimoine français, et d'amélioration de l'accès à la littérature destinée aux mathématiciens. Les termes de la négociation avec les auteurs et les éditeurs, tenant compte des règles du droit d'auteur, ont été présentés, puis des aspects plus techniques liés à l'adoption de standards non propriétaires, au contrôle de la qualité, à la gestion des métadonnées et à la création de liens de diverses natures au sein du web. Ce projet, qui doit se dérouler en plusieurs phases, est mené parallèlement à d'autres projets de bibliothèques numériques réalisés en collaboration avec de multiples institutions européennes, américaines et françaises. L'objectif est de procurer dans le domaine mathématique, science cumulative, un accès aisé et à long terme à une bibliothèque de qualité scientifique, d'adopter des modèles économiques raisonnables, une structuration logique et des liens de référence de plus en plus fins (voire, à terme, jusqu'au théorème).

\footnotetext{
${ }^{6}$ Pour en savoir plus sur I'Initiative de Budapest : 〈www.soros.org/openaccess/fr/index.shtml>

${ }^{7}$ Fondation créée par Georges Soros, I'Open society Institute est à l'origine de diverses actions, non limitées au domaine scientifique.,
} 
- Le Centre pour la communication scientifique directe. Créé au sein du CNRS, le CCSD est une approche " archives ouvertes » à vocation internationale, favorisant l'auto-archivage. Il propose divers services: HAL, serveur d'articles alimenté par le chercheur lui-même, adopte les mêmes règles et les mêmes formats que la base $\mathrm{ArXiv}^{8}$ (I'alimentation se fait concurremment dans les deux bases), mais procure plus de métadonnées, couvre plus de disciplines et propose un système de "vue privée», "zone tampon qui permet de reconnaître certains articles»; TEL, serveur de thèses multidisciplinaire, alimenté par l'auteur ou à partir de bases de données déjà contrôlées, propose également un service de DSI ; l'extension MetHal pour les notices.

Ont aussi été présentées les collaborations engagées avec divers instituts, les règles de soumission dans les serveurs du CCSD et les formats de documents adoptés, ainsi que les protocoles d'exports et les technologies utilisées. Le système doit avoir à terme une plus grande couverture pluridisciplinaire, assurer une rétroconversion des thèses et des articles, une meilleure articulation par des liens croisés. L'accent a également été mis sur le souci d'un archivage à long terme et d'une visibilité internationale.

- SPARC Europe. Cette initiative a été lancée en juillet 2001. Comme SPARC (Scholarly Publishing and Academic Ressources Coalition), créée précédemment aux États-Unis, elle veut mener diverses actions en faveur de la recherche et des bibliothèques, mais dans une optique européenne. Comme elle également, elle veut offrir aux auteurs, aux lecteurs et aux bibliothèques des solutions alternatives aux revues dont les prix se sont accrus de façon considérable. Plusieurs projets, liés aux opportunités offertes par l'environnement numérique, ont été présentés. Ils ont pour objectif, par le biais de diverses actions menées en partenariat, de réduire les coûts liés à la logistique, de trouver des moyens de financement, de favoriser un système de paiement lié à la diffusion et non à l'accès, d'assurer la certification des publications et de mener diverses actions de sensibilisation ${ }^{9}$ au libre accès.

- Figaro. La Federated Initiative of GAp and ROquade est un projet européen qui veut fournir un modèle d'édition universitaire numérique, encourageant le libre accès aux publications produites. Ce modèle présente des composantes économique et organisationnelle, mais c'est surtout sur les aspects techniques, qui ont des impacts politiques et économiques, que l'accent a été mis. Notamment - ce système ayant adopté le désormais classique protocole OAI - sur des aspects techniques plus rarement évoqués, qui utilisent pleinement les possibilités du numérique pour la définition de ce modèle d'édition fédératif et ouvert. Celui-ci veut servir de "support pour des documents standards édités en format XML et auX outils d'écriture qui y sont liés, permettre une utilisation partagée d'un moteur web de contrôle des flux de travaux et servir de support pour des méthodes génériques d'authentification et d'autorisation et pour des fonctions de gestion de contenu hétérogènes et distribuées, comprenant des technologies de pointage continu et des services d'impression sur demande». Le coordinateur de ce projet conclut en rappelant que l'adoption d'une technique donnée n'est pas neutre, que les initiatives purement politiques sont dangereuses et que la maîtrise du contenu doit être maintenue.

- Le projet E-BioSci. Il s'agit d'un projet de plate-forme européenne pour l'accès et la recherche de documents en texte intégral et de données factuelles dans le domaine des sciences du vivant. La conception de ce portail est également aidée financièrement par les instances européennes. Là encore, l'accent a été mis sur les outils qui doivent pouvoir saisir non seulement

\footnotetext{
${ }^{8}$ ArXiv est sans doute le premier site ayant hébergé des publications en libre accès, au départ dans le domaine de la physique. 〈http://xxx.lanl.gov>

${ }^{9}$ On ne citera que le site Create change 〈www.createchange.org〉, guide destiné aux universités et aux bibliothécaires.
} 
la masse d'informations proposées dans différentes bases éparpillées dans plusieurs centres de recherche, mais également comprendre la complexité du langage naturel, reconnaître sans ambiguïté des gènes ou d'autres symboles utilisés par les scientifiques. Développé avec divers partenaires européens, E-BioSci propose déjà un prototype qui permet une recherche intelligente en texte intégral, concurremment dans plusieurs archives en libre accès, en utilisant un métalangage. Les versions suivantes permettront de réaliser des représentations graphiques des relations sémantiques entre les documents et les informations obtenus après une recherche dans les différents éléments de ce portail.

\section{Une opportunité pour les pays en développement}

En introduction à une table ronde consacrée aux pays en développement, Kay Raseroka, présidente élue de I'IFLA, souligne que le libre accès peut apporter des solutions à la rareté des ressources et à l'insuffisance d'infrastructures, et stopper l'émigration des compétences. Actuellement la fracture Nord/Sud est énorme, la recherche dans les pays du Sud n'est ni connue ni même reconnue, et le cercle vicieux dans lequel on est installé pousse les chercheurs à émigrer. L'édition électronique permet d'accroître l'impact des revues et la visibilité de la recherche des pays du Sud, mais aussi de susciter des actions en partenariat dans le monde scientifique et d'enclencher une spirale positive. L'étape suivante consiste à rendre ces documents disponibles gratuitement, à combler non seulement la fracture Nord/Sud mais aussi

celles qui existent entre les pays du Sud. À cette fin, l'organisation d'ateliers de sensibilisation au libre accès dans les pays en développement s'impose.

Le ministère des Affaires étrangères et l'Agence universitaire de la francophonie ont engagé, en partenariat avec les PED, plusieurs programmes et actions pour lutter contre la fracture numérique, favoriser l'accès au savoir et valoriser la recherche du Sud. Lorsqu'on aborde le problème de l'IST, il est important de tenir compte de la situation économique d'un pays donné et de ses composantes culturelles. Il faut se souvenir que la mobilité est nécessaire mais qu'elle ne doit pas se traduire par un exode durable. Pour s'opposer à la marchandisation de l'éducation, il est nécessaire de veiller à ce que les résultats de la recherche puissent être réutilisés, non gratuitement au risque de dévaloriser l'information, mais, par le biais de subventions, à des coûts proportionnels au niveau de vie d'un professionnel vivant dans un pays du Sud. L'accès au contenu, par le biais notamment du libre accès, ne correspond qu'à un maillon de la chaîne en faveur de la diversité culturelle qu'il faut préserver.

Il est important de maintenir dans les pays en développement une activité scientifique dans le domaine médical. Pour y contribuer, I'Organisation mondiale de la santé a développé des programmes qui facilitent l'accès au document en proposant des coûts réduits et un libre accès, et en veillant à minimiser le temps de connexion, autre élément important du coût. Une attention particulière doit être portée à la pérennité des actions menées, souvent en partenariat. S'il est souvent difficile de mesurer concrètement les apports de ces programmes, on a pu noter que le taux de participation des chercheurs des pays du Sud aux congrès médicaux était de plus en plus forte.

L'Association internationale pour la promotion de la coopération avec les scientifiques dans les nouveaux États indépendants de l'ancienne Union soviétique (INTAS) œuvre en faveur des chercheurs des pays en développement, y compris par le biais des bibliothèques électroniques. Il est reconnu que le libre accès et la mise à disposition des publications gratuite ou à très bas prix permettent d'augmenter la participation des chercheurs à des projets scientifiques. Mais ce ne sont que des étapes intermédiaires car le renforcement des capacités pour assurer la pérennité d'une situation plus favorable a plus d'impact. 
À cet effet, des actions de formation doivent être proposées, y compris pour des bibliothécaires et des informaticiens. Elles doivent être envisagées dans un sens très large et comprendre l'insertion des individus ou des institutions dans des réseaux. La mobilité, indispensable, ne doit rester qu'une étape enrichissante dans un cursus professionnel. Les actions menées doivent respecter la situation économique et la diversité culturelle, et les pays du Sud doivent en être de véritables acteurs. Il est urgent de créer les conditions pour que ces pays puissent développer eux-mêmes leurs capacités bien que, dans beaucoup d'entre eux qui connaissent des crises graves, l'action en faveur de la recherche n'y soit pas considérée comme une priorité.

Vers de nouvelles études et de nouvelles expérimentations...

Le libre accès est un moyen de se réapproprier les informations produites, de faire face à la croissance exponentielle de l'information, mais il ne doit pas remplacer le système de diffusion traditionnel. Il offre des solutions nouvelles, mieux adaptées aux besoins. Il soulève néanmoins des questions liées à l'évaluation des articles ou à l'archivage à long terme. Il faut, selon Geneviève Berger, directrice générale du CNRS, poursuivre la réflexion pour mettre au point des systèmes plus performants, parallèlement à une autre sur l'évolution des pratiques d'accès à I'IST.

Christian Brechot, directeur de l'INSERM, insiste enfin sur l'évolution notée depuis 1998 et les premières discussions avec la maison d'édition Elsevier. Il souligne que les archives ne représentent pas une simple liste de publications mais un ensemble de connaissances, et que l'accroissement de l'accès à l'information scientifique et technique peut avoir une synergie très forte. Il appelle de ses vœux une réflexion qui implique l'ensemble des organismes de recherche français sur les systèmes d'évaluation des chercheurs et des activités scientifiques, ainsi que sur la pérennité du système. Une réflexion fondée sur trois principes: la conservation de l'antériorité et de la propriété intellectuelle, la qualité des résultats, la meilleure visibilité possible.

Michèle Battisti

michele.battisti@adbs.fr

\section{Le programme du séminaire}

Ce séminaire a eu lieu à Paris les 23 et 24 janvier 2003. Il était organisé par le ministère délégué à la Recherche et aux Nouvelles Technologies, l'Institut national de la santé et de la recherche médicale (INSERM), le Centre national de la recherche scientifique (CNRS), I'Institut de l'information scientifique et technique (INIST) et l'International Council for Scientific and Technical Information (ICSTI), avec le soutien de I'ICSU/CODATA (International Council for Science / Committee on Data for Science and Technology). Il a réuni les vingt-huit intervenants suivants.

- Allocution d'ouverture, par Bernard Bigot, directeur de cabinet de madame la ministre déléguée à la Recherche et aux Nouvelles Technologies

\section{Introduction et vue d'ensemble}

- Christine Deschamps, présidente de la Fédération internationale des associations de bibliothécaires et des bibliothèques (IFLA)

- Kurt Molholm, président de l'ICSTI

- Pierre Oudet, professeur, conseiller en IST à l'INSERM 
- Dominique Wolton, directeur de recherche au CNRS, sociologue, écrivain

- Nouvelle intermédiation dans la République des Sciences: de la propriété intellectuelle à la communauté des biens intellectuels, par Paul F. Uhlir, directeur du Bureau des programmes internationaux en IST et du Comité national des États Unis de CODATA (Committee on Data for Science and Technology), membre de l'Académie nationale des Sciences et du Conseil national de la recherche des États Unis

- Rapport sur l'évolution du libre accès et ses conséquences, par Jack Franklin, directeur de ASFRA bv, Edam, Pays-Bas

Le libre accès: quelles conséquences pour la diffusion de I'IST?

- Pieter Bolman, directeur des relations STM, Elsevier Science

- Sally Morris, secrétaire générale de l'Association of Learned and Professional Society Publishers (ALPSP)

- Andy Powell, directeur adjoint de l'UK Office for Library Networking (UKOLN), Université de Bath, membre du Comité technique Open Archives Initiative (OAI)

- Geoffrey Bilder, responsable en chef des technologies d'INGENTA

- Jan Velterop, éditeur de BioMed Central

- Elmar Mittler, président du Groupe de Francfort, ancien président de la Ligue des bibliothèques européennes de recherche (LIBER) et directeur de la bibliothèque de I'Université de Göttingen

- Thomas Dreier, professeur à la Faculté de droit de l'Université de New York et directeur de I'Institut du droit de l'information de l'Université de Karlsruhe

Le libre accès : quels enjeux pour la communauté scientifique?

- Laurent Guillopé, professeur à l'Université de Nantes, directeur adjoint de la cellule MathDoc du CNRS

- Daniel Charnay, directeur adjoint du Centre pour la communication scientifique directe (CCSD) du CNRS

- Stevan Harnad, professeur de sciences cognitives à I'Université de Southampton et à I'Université du Québec à Montréal

Le libre accès : quels enjeux pour les pays en développement?

- Kay Raseroka, présidente élue de la Fédération internationale des associations de bibliothécaires et des bibliothèques (IFLA)

- Barbara Aronson, responsable du projet HINARI (Interréseau-Santé Initiative d'Accès aux Recherches) à l'Organisation mondiale de la santé (OMS)

- Georges Malamoud, de la Direction de la planification et de la prospective de l'Agence universitaire de la francophonie (AUF)

- Manfred Spiesberger, responsable du projet Action pour une infrastructure de bibliothèque numérique à l'Association internationale pour la promotion de la coopération avec les scientifiques dans les nouveaux États indépendants de l'ancienne Union soviétique (INTAS)

- Jean-Jacques Pierrat, chef du bureau du Développement technologique, de l'Information et de la Culture scientifique à la sous-direction de la Recherche du ministère des Affaires étrangères 
- SPARC (Scholarly Publishing and Academic Resources Coalition), par David Prosser, directeur de SPARC Europe, Oxford

- L'Initiative de Budapest pour l'accès ouvert, par Jean-Claude Guédon, professeur à l'Université de Montréal, membre de la commission de l'information de l'Open Society Institute

- FIGARO (European Academic Digital Publishing Initiative), par Stefan Gradmann, coordinateur technique du projet, chef de l'unité Bibliothèque virtuelle, Université de Hambourg

- E-BIOSCI (Portail européen d'accès à l'information factuelle et en texte intégral en sciences du vivant), par Les Grivell, responsable du projet

Allocutions de clôture et perspectives d'avenir

- Geneviève Berger, directrice générale du Centre national de la recherche scientifique (CNRS)

- Christian Brechot, directeur général de I'Institut national de la santé et de la recherche médicale (INSERM)

\section{Pour en savoir plus}

- Le libre accès à l'information scientifique et technique, rubrique proposée par l'INIST sur son site. On y trouvera les enregistrements vidéo de toutes les interventions faites lors de ce séminaire ainsi que de nombreux documents sur le même sujet, apportés régulièrement en complément. 〈www.inist.fr/openaccess>

- La communication scientifique, répertoire réalisé par Hélène Bosc en collaboration avec Simone Jérôme et Jean-Philippe Schmitt. 〈www.tours.inra.fr/tours/doc/comsci.htm〉

- Les archives ouvertes dans la communication scientifique, répertoire réalisé par Ghislaine Chartron. 〈web.ccr.jussieu.fr/urfist/archives-ouvertes.html>

- Un exemple d'archives ouvertes dans le domaine de l'information-documentation: @rchiveSIC

- archives en sciences de l'information et de la communication. 〈archiveSIC.ccsd.cnrs.fr〉

- Sur le site de I'Initiative de Budapest pour l'accès ouvert aux publications, on trouvera une FAQ qui peut s'avérer utile, malgré quelques répétitions et maladresses de traduction.

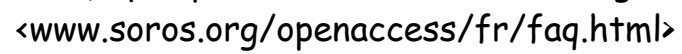

- Les travaux de ce séminaire devaient se poursuivre par une autre manifestation organisée par CODATA, les 10 et 11 mars 2003 dans les locaux de I'Unesco, sur l'accès libre et le domaine public en matière de données et d'informations numériques scientifiques. Elle devait mettre davantage l'accent sur les apports de ce mouvement pour les pays en développement. <www.codata.org/03march-program.htm>

\section{Précisions terminologiques}

Les termes utilisés dans ce compte rendu correspondent souvent à des traductions d'expressions anglaises et peuvent quelquefois prêter à confusion.

- L'expression «archives ouvertes» (open archives) où le terme « archives » doit être entendu au sens d'archives vivantes et où le qualificatif « ouvert»s'oppose à la notion «propriétaire » et renvoie à l'idée de consultation libre et gratuite. Le qualificatif « ouvert» a aussi une connotation technique et signifie qu'il y a une ouverture de l'architecture technique par des protocoles communs facilitant l'accès aux contenus. Les «archives ouvertes» sont souvent 
associées à l'Open Archives Initiative, qui préconise l'interopérabilité des documents par des normes qui peuvent être appliquées à tout type d'archives, y compris à des archives verrouillées.

- L'expression «libre accès» (open access) implique uniquement un accès non verrouillé, une notion différente de la notion de ressources gratuites. Si le terme « ouvert» peut ici avoir une signification différente du terme «libre», on rappellera aussi que le mot free, «libre», ne se traduit pas toujours par « gratuit». Libre accès ne veut pas forcément dire accès gratuit, mais seulement libre consultation (voir, sur ce point, les ceuvres dites «libres de droit», terme qui prête également à confusion car en pratique de nombreuses clauses en limitent les exploitations possibles).

\section{Sites web des organisations citées}

ALPSP : www.alpsp.org>

BioMed Central : 〈www.biomedcentral.com>

CCSD : 〈www.ccsd.cnrs.fr $\rangle$

CODATA : 〈www.codata.org $>$

E-Bio-Sci : 〈www.e-bisoci.org>

Figaro: <www.figaro-europe.net/index2.html?news.html

ICSTI : 〈www.icsti.org>

IFLA : 〈www.ifla.org>

Ingenta : <www.ingenta.com>

Initiative de Budapest : 〈www.soros.org/openaccess/fr/index.shtml>

INTAS : <www.intas.be>

LIBER : 〈www.kb.dk/guests/liber/intl/liber/francais/about>

NUMDAM : 〈www.numdam.org>

Open Archives Initiative (OAI) : 〈www.openarchive.org>

Open Society Institute : 〈www.soros.org〉

SPARC Europe : 〈www.sparceurope.org> 